\title{
Cosmetic surgery in L-space homology spheres
}

\author{
ZHONGTAO WU
}

Let $K$ be a nontrivial knot in $S^{3}$, and let $r$ and $r^{\prime}$ be two distinct rational numbers of same sign. We prove that there is no orientation-preserving homeomorphism between the manifolds $S_{r}^{3}(K)$ and $S_{r^{\prime}}^{3}(K)$. We further generalize this uniqueness result to knots in arbitrary L-space homology spheres.

$57 \mathrm{M} 25,57 \mathrm{M} 27$

\section{Introduction}

It has been known for a long time that every closed connected orientable three-manifold is obtained by surgery on a link in $S^{3}$. However, a classification of three-manifolds in terms of this surgery construction has remained elusive, due primarily to the lack of uniqueness of the surgery description.

In particular, let $K$ be a framed knot in a closed oriented three-manifold $Y$. For a rational number $r$, let $Y_{r}(K)$ be the manifold obtained by Dehn surgery along $K$ with slope $r$. Two surgeries along $K$ with distinct slopes $r$ and $r^{\prime}$ are called equivalent if there exists an orientation-preserving homeomorphism of the complement of $K$ taking one slope to the other; and they are called truly cosmetic if there exists an orientation-preserving homeomorphism between $Y_{r}(K)$ and $Y_{r^{\prime}}(K)$.

When $K=U$ is the unknot in $S^{3}$, there are truly cosmetic surgeries: $S_{p / q}^{3}(U) \cong$ $S_{p / p+q}^{3}(U)$, and $S_{p / q_{1}}^{3} \cong S_{p / q_{2}}^{3}(U)$ when $q_{1} q_{2} \equiv 1(\bmod p)$. While $p / q$ and $p /(p+q)$ are equivalent slopes, $p / q_{1}$ and $p / q_{2}$ as above are usually not. By contrast, there are no known truly cosmetic surgeries on a nontrivial knot. Indeed, it is one of the outstanding problems conjectured in Kirby's problem list, Problem 1.81(1):

Conjecture 1.1 (Cosmetic Surgery Conjecture [4]) Two surgeries on a nontrivial knot with nonequivalent slopes are never truly cosmetic.

Lackenby [6] has showed that under general conditions on a null-homotopic knot $K \subset Y$, there are at most finitely many truly cosmetic surgeries: Consider two surgered 
manifolds $Y_{p / q}(K)$ and $Y_{p^{\prime} / q^{\prime}}^{\prime}\left(K^{\prime}\right)$. If $Y$ and $Y^{\prime}$ are distinct, or $K$ and $K^{\prime}$ are distinct, or $p / q \neq p^{\prime} / q^{\prime}$, then $Y_{p / q}(K) \not Y_{p^{\prime} / q^{\prime}}^{\prime}\left(K^{\prime}\right)$ for a sufficiently large $|q|$.

Techniques from Heegaard Floer homology were brought into the study of the cosmetic surgery problem by Ozsváth and Szabó in [15]. In particular, they proved the following important results: if $S_{r}^{3}(K) \cong \pm S_{r^{\prime}}^{3}(K)$, then either $S_{r}^{3}(K)$ is an L-space or $r$ and $r^{\prime}$ have opposite signs (See Definition 1.3 for the definition of $L$-space). Combining this with number theoretical methods, Wang was able to prove the conjecture for all genus-one knots in $S^{3}$ [18]. Ni, in another direction, used a twisted version of the Heegaard Floer homology to prove the case when $\mathrm{K}$ is a null-homologous knot in a closed three-manifold $Y$ that contains a nonseparating sphere [7].

In [1], Boyer and Lines proved, among other things, the cosmetic surgery conjecture for any knot $K$ with $\Delta_{K}^{\prime \prime}(1) \neq 0$. Based on their results and by introducing the techniques from [15], we prove the following uniqueness theorem.

Theorem 1.2 Let $r$ and $r^{\prime}$ be two distinct rational numbers with $r r^{\prime}>0$, and let $K$ be a nontrivial knot in $S^{3}$. Then $S_{r}^{3}(K) \not S_{r^{\prime}}^{3}(K)$.

An immediate implication of this theorem is that an oriented manifold can be obtained from at most TWO surgeries on a nontrivial knot. One should also compare it with Gordon and Luecke's knot complement theorem [3], which asserts that $S_{r}^{3}(K) \not S^{3}=$ $S_{\infty}^{3}(K)$. Many results of this type, including this knot complement theorem, were proved by Kronheimer, Mrowka, Ozsváth and Szabó [5] and Ozsváth and Szabó [15], purely based on Floer theory.

Definition 1.3 A three-manifold $Y$ is called an L-space if $Y$ is a rational homology sphere and $\widehat{H F}(Y, \mathfrak{s}) \cong \mathbb{Z}$ for each $\operatorname{Spin}^{c}$ structure $\mathfrak{s}$ on $Y$.

An L-space is a natural generalization of the notion of lens space in Heegaard Floer homology. An $L$-space homology sphere is an L-space with $\widehat{H F}(Y) \cong \mathbb{Z}$. This is a class of three-manifolds with the simplest $\widehat{H F}$, whose members include $S^{3}$, the Poincaré sphere $\Sigma(2,3,5)$, the Poincaré sphere with opposite orientation $-\Sigma(2,3,5)$, and their connected sums. Theorem 1.2 is also true for knots in arbitrary L-space homology spheres.

Theorem 1.4 Let $r$ and $r^{\prime}$ be two distinct rational numbers with $r r^{\prime}>0$, and let $K$ be a nontrivial knot in an $L$-space homology sphere $Y$. Then $Y_{r}(K) \nRightarrow Y_{r^{\prime}}(K)$.

Our proof of the above theorems involves a simultaneous application of the CassonWalker invariant, the Casson-Gordon invariant and Heegaard Floer homology. This 
argument can be potentially generalized to knots in lens spaces and L-space rational homology spheres, and it is still a work in progress. Various other partial results and improvements may also be obtained, if we impose additional conditions on the knot $K$, such as its genus, or on the surgery coefficients.

The paper is organized as follows. In Section 2, we sketch some background in the Casson-Walker and Casson-Gordon invariants and present explicit surgery formulae for the two invariants. In Section 3, we recall some preliminaries in Heegaard Floer homology and make a few slight generalizations on the setup, mostly from $S^{3}$ to L-space homology spheres. Finally in Section 4, we put everything together and prove our main results.

Acknowledgments I am grateful to my advisor, Zoltán Szabó, for suggesting the problem and spending countless time in guiding and advising. Thanks in addition to Steven Boyer, David Gabai, Joshua Greene, Yi Ni and JiaJun Wang for many helpful discussions at various points. Finally, I would like to acknowledge Sam Lewallen for sharing the reference of Boyer and Lines [1].

\section{Preliminaries in classical three-manifold invariants}

We sketch in this section the definition and basic properties of two classical threemanifold invariants: the Casson-Walker invariant and the Casson-Gordon invariant.

\subsection{Casson-Walker invariant}

The Casson invariant is one of the many invariants of a closed three-manifold $Y$ that can be obtained by studying representations of its fundamental group in a certain nonabelian group $G$. Roughly speaking, the Casson invariant of an integral homology sphere $Y$ is obtained by counting representations of $\pi_{1}(Y)$ in $G=\mathrm{SU}(2)$. The geometric structures used to obtain a topological invariant is a Heegaard splitting of $Y$ and the symplectic geometry associated with it. An alternative gauge-theoretical approach uses flat bundles together with a Riemannian metric on $Y$ and leads to a refinement of the Casson invariant, the Floer homology.

Casson's SU(2) intersection theory was later extended by Walker to include reducible representations, who generalized the invariant to rational homology spheres. Most remarkably, Walker's invariant admits a purely combinatorial definition in terms of surgery presentations. The existence and uniqueness of this invariant together with basic properties are given by the following theorem in Walker [17]. 
Theorem 2.1 There exists a unique invariant $\lambda$, satisfying the following properties:

(1) $\lambda$ coincides with Casson's invariant on integral homology sphere.

(2) $\lambda(-Y)=-\lambda(Y)$ where $-Y$ stands for $Y$ with reversed orientation.

(3) $\lambda\left(Y_{1} \# Y_{2}\right)=\lambda\left(Y_{1}\right)+\lambda\left(Y_{2}\right)$ for two rational homology spheres $Y_{1}$ and $Y_{2}$.

(4) The number $12 \cdot\left|H_{1}(Y, \mathbb{Z})\right| \cdot \lambda(Y)$ is an integer for any rational homology sphere.

(5) Let $k$ be a knot in a rational homology sphere $Y, K$ its exterior, and $l \in \partial K$ a longitude. Then, $\lambda$ satisfies the surgery formula

$$
\lambda\left(K_{a}\right)=\lambda\left(K_{b}\right)+\tau(a, b ; l)+\frac{\langle a, b\rangle}{\langle a, l\rangle\langle b, l\rangle} \cdot \Delta_{K}^{\prime \prime}(1)
$$

for primitive $a, b \in H_{1}(\partial K, \mathbb{Z})$ such that $\langle a, l\rangle \neq 0$ and $\langle b, l\rangle \neq 0$

Here, the brackets $\langle$,$\rangle denote the intersection pairing H_{1}(\partial K, \mathbb{Z}) \otimes H_{1}(\partial K, \mathbb{Z}) \longrightarrow \mathbb{Z}$. Given a longitude $l$, choose a basis $x, y$ of $H_{1}(\partial K, \mathbb{Z})$ such that $\langle x, y\rangle=1$ and $l=d y$ for some $d \in \mathbb{Z}$. Then

$$
\tau(a, b ; l)=-s(\langle x, a\rangle,\langle y, a\rangle)+s(\langle x, b\rangle,\langle y, b\rangle)+\frac{d^{2}-1}{12} \cdot \frac{\langle a, b\rangle}{\langle a, l\rangle\langle b, l\rangle} .
$$

Definition 2.2 Given two coprime numbers $p$ and $q$, the Dedekind sum $s(q, p)$ is defined by

where

$$
\begin{aligned}
s(q, p): & =\operatorname{sign}(p) \cdot \sum_{k=1}^{|p|-1}\left(\left(\frac{k}{p}\right)\right)\left(\left(\frac{k q}{p}\right)\right), \\
((x)) & = \begin{cases}x-[x]-\frac{1}{2} & \text { if } x \notin \mathbb{Z}, \\
0 & \text { if } x \in \mathbb{Z} .\end{cases}
\end{aligned}
$$

When $p, q>0$, write $p / q$ as a continued fraction

$$
\frac{p}{q}=\left[a_{1}, \ldots, a_{n}\right]=a_{1}-\frac{1}{a_{2}-\frac{1}{a_{3}-\cdots}} .
$$

An equivalent definition of the Dedekind sum is given by

$$
s(q, p):=\frac{1}{12}\left(\frac{q}{p}+\frac{q^{\prime}}{p}+\sum_{i=1}^{n}\left(a_{i}-3\right)\right)
$$

where $0<q^{\prime}<p$ is the unique integer such that $q q^{\prime} \equiv 1(\bmod p)$.

The surgery formula is much simplified when applied to null-homologous knots in rational homology spheres. 
Proposition 2.3 Let $K$ be a null-homologous knot in a rational homology threesphere $Y$, and let $L(p, q)$ be the lens space obtained by $(p / q)$-surgery on the unknot in $S^{3}$. Then

$$
\lambda\left(Y_{p / q}(K)\right)=\lambda(Y)+\lambda(L(p, q))-\frac{q}{p} \Delta_{K}^{\prime \prime}(1) .
$$

Be aware that our definition of the Casson-Walker invariant may differ from that of various other references by a factor of 2 . Our normalization is made for the convenience of the following fact among other things.

Proposition 2.4 For a lens space $L(p, q), \lambda(L(p, q))=s(q, p)$.

\subsection{Casson-Gordon invariant}

We would like to begin our discussions of the Casson-Gordon invariant by quoting the following $G$-signature theorem for closed four-manifolds by Rokhlin.

Theorem 2.5 ( $G$-Signature Theorem) Suppose $\pi: \tilde{X} \rightarrow X$ is an $m$-fold cyclic cover of closed four-manifolds $\tilde{X}$ branched over a properly embedded surface $F$ in $X$. Then,

$$
\operatorname{sig}(\tilde{X})=m \cdot \operatorname{sig}(X)-[F]^{2} \cdot \frac{m^{2}-1}{3 m} .
$$

Consider a closed oriented three-manifold $Y$ with $H_{1}(Y ; \mathbb{Z})=\mathbb{Z}_{m}$. It has a unique $m$-fold cyclic cover $\tilde{Y} \rightarrow Y$. Pick up an $m$-fold cyclic branched covering of fourmanifold $\widetilde{W} \rightarrow W$, branched over a properly embedded surface $F$ in $W$, such that $\partial(\widetilde{W} \rightarrow W)=(\widetilde{Y} \rightarrow Y)$. The existence of such $(W, F)$ follows from [2, Lemma 2.2].

Definition 2.6 Define the total Casson-Gordon invariant of $Y$ by

$$
\tau(Y)=m \cdot \operatorname{sig}(W)-\operatorname{sig}(\widetilde{W})-[F]^{2} \cdot \frac{m^{2}-1}{3 m} .
$$

It is a standard argument to see the independence of the definition on the choice of the four manifolds cover $\widetilde{W} \rightarrow W$. Suppose $\widetilde{W}^{\prime} \rightarrow W^{\prime}$ is another cover that bounds $\tilde{Y} \rightarrow Y$, then we can construct a branched cover $-\widetilde{W}^{\prime} \cup \widetilde{M} \widetilde{W} \rightarrow-W^{\prime} \cup_{M} W$ of closed four-manifold. It follows readily from Novikov additivity and the $G$-signature Theorem that the invariant is well defined.

Definition 2.7 Let $K$ be a knot in an integral homology sphere $Y$ and $m \neq 0$. The generalized signature function $\sigma_{K}(\xi)$ is the signature of the matrix $A(\xi):=$ $(1-\bar{\xi}) A+(1-\xi) A^{T}$ for a Seifert matrix $A$ of $K$, where $|\xi|=1$. 
A surgery formula for the total Casson-Gordon invariant was established by Boyer and Lines [1].

Proposition 2.8 Let $K$ be a knot in an integral homology sphere $Y$, then

$$
\tau\left(Y_{p / q}(K)\right)=\tau(L(p, q))-\sigma(K, p),
$$

where $\sigma(K, p)=\sum_{r=1}^{p-1} \sigma_{K}\left(e^{2 i \pi r / p}\right)$.

Quite amazingly, the total Casson-Gordon invariant of the lens space $L(p, q)$ is also related to the Dedekind sum.

Proposition 2.9 For a lens space $L(p, q), \tau(L(p, q))=-4 p \cdot s(q, p)$.

\section{Preliminaries in Heegaard Floer homology}

Heegaard Floer homology is an invariant for closed three manifolds Y (see Ozsváth and Szabó $[12 ; 11])$. The invariant, denoted $H F^{\circ}(Y)$, is the homology of a chain complex whose generators have a combinatorial definition, and whose boundary operator counts certain pseudo-holomorphic disks in associated spaces.

In Ozsváth and Szabó [10] and Rasmussen [16], a closely related invariant is defined for null-homologous knots $K$ in a closed, oriented three-manifold $Y$, taking the form of an induced filtration on the Heegaard Floer complex of $Y$. The filtered chain homotopy type of this complex is a knot invariant, known as "knot Floer homology". Given an integer $n$, let $Y_{n}(K)$ denote the three-manifold obtained by $n$-framed surgery on $Y$ along $K$. When $n$ is sufficiently large, there is an immediate relationship between the knot Floer homology of $K$ and the Heegaard Floer homology of $Y_{n}(K)$; see [10, Section 4]. We next review the general case for an arbitrary integer or rational surgery on an integral homology sphere $Y$ which was developed by Ozsváth and Szabó in [14; 15].

\subsection{Rational surgery formulas}

Knot Floer homology associates to a knot $K$ a $\mathbb{Z} \oplus \mathbb{Z}$-filtered $\mathbb{Z}[U]$-complex $C=$ $C F K^{\infty}(Y, K)$, generated over $\mathbb{Z}$ by a set $X$ equipped with a function $\mathcal{F}: X \rightarrow \mathbb{Z} \oplus \mathbb{Z}$ with the property that, if $\mathcal{F}(\mathbf{x})=(i, j)$, then $\mathcal{F}(U \cdot \mathbf{x})=(i-1, j-1)$ and $\mathcal{F}(\mathbf{y}) \leq \mathcal{F}(\mathbf{x})$ for all $\mathbf{y}$ having nonzero coefficient in $\partial \mathbf{x}$.

Let $S$ be a region in the plane with the property that $(i, j) \in S$ implies $(i+1, j)$, $(i, j+1) \in S$, and let $C\{S\}$ be the natural quotient complex of $C$ generated by $\mathbf{x}$ with $\mathcal{F}(\mathbf{x}) \in S$. For an integer $s$, we define $A_{s}^{+}(K):=C\{\max (i, j-s) \geq 0\}$ and 
$B^{+}(K):=C\{i \geq 0\}$. There are two canonical chain maps $v_{s}^{+}: A_{s}^{+} \rightarrow B^{+}$and $h_{s}^{+}: A_{s}^{+} \rightarrow B^{+}$. The map $v_{s}^{+}$is projection onto $C\{i \geq 0\}$, while $h_{s}^{+}$is projection onto $C\{j \geq s\}$, followed by the identification with $C\{j \geq 0\}$, followed by the chain homotopy equivalence from $C\{j \geq 0\}$ to $C\{i \geq 0\}$.

Now fix a surgery slope $p / q$ and suppose $q>0$. Consider the two chain complexes

$$
\mathbb{A}^{+}=\bigoplus_{t \in \mathbb{Z}}\left(t, A_{\lfloor t / q\rfloor}^{+}\right), \quad \mathbb{B}^{+}=\bigoplus_{t \in \mathbb{Z}}\left(t, B^{+}\right),
$$

where $\lfloor x\rfloor$ is the greatest integer not bigger than $x$. An element of $\mathbb{A}^{+}$could be written as $\left\{\left(t, a_{t}\right)\right\}_{t \in \mathbb{Z}}$ with $a_{t} \in A_{\lfloor t / q\rfloor}^{+}$. Define a chain map $D_{p / q}^{+}: \mathbb{A}^{+} \rightarrow \mathbb{B}^{+}$by

$$
D_{p / q}^{+}\left\{\left(t, a_{t}\right)\right\}=\left\{\left(t, b_{t}\right)\right\},
$$

where

$$
b_{t}=v^{+}\left(a_{t}\right)+h^{+}\left(a_{t-p}\right) .
$$

Let $\mathbb{X}_{p / q}^{+}(K)$ be the mapping cone of $D_{p / q}^{+}$.

Note that $\mathbb{X}_{p / q}^{+}$naturally splits into the direction sum of $p$ subcomplexes

$$
\mathbb{X}_{p / q}^{+}=\bigoplus_{i=0}^{p-1} \mathbb{X}_{i, p / q}^{+}
$$

where $\mathbb{X}_{i, p / q}^{+}$is the subcomplex of $\mathbb{X}_{p / q}^{+}$containing all $A_{t}^{+}$and $B_{t}^{+}$with $t \equiv i \bmod p$.

The Heegaard Floer homology of a $p / q$ surgered manifold is determined by the mapping cone $\mathbb{X}_{p / q}^{+}$according to the following theorem of Ozsváth and Szabó.

Theorem 3.1 (Ozsváth and Szabó [15]) Let $K \subset Y$ be a nullhomologous knot and $p, q$ a pair of coprime integers. Then, for each $i \in \mathbb{Z} / p \mathbb{Z}$, there is a relatively graded isomorphism of groups

$$
H F^{+}\left(Y_{p / q}(K), \mathfrak{s}_{i}\right) \cong H_{*}\left(\mathbb{X}_{i, p / q}^{+}\right),
$$

where $\mathfrak{s}_{i}$ is the $\operatorname{Spin}^{c}$ structure corresponding to $i \in \mathbb{Z} / p \mathbb{Z}$.

An analogous surgery formula for $\widehat{H F}$ can be obtained if we restrict the preceding formulation to its "boundary". Let $\widehat{A}_{S}(K):=C_{K}\{\max (i, j-s)=0\}$ and $B^{+}(K):=$ $C\{i=0\}$, and let $\hat{v}, \hat{h}$ be the induced projection maps. We have the corresponding mapping cone $\widehat{\mathbb{X}}_{i, p / q}(K)$. 
Theorem 3.2 (Ozsváth and Szabó [15]) Let $K \subset Y$ be a nullhomologous knot and $p, q$ a pair of coprime integers. Then, for each $i \in \mathbb{Z} / p \mathbb{Z}$, there is a relatively graded isomorphism of groups

$$
\widehat{H F}\left(Y_{p / q}(K), \mathfrak{s}_{i}\right) \cong H_{*}\left(\widehat{\mathbb{X}}_{i, p / q}\right),
$$

where $\mathfrak{s}_{i}$ is the $\operatorname{Spin}^{c}$ structure corresponding to $i \in \mathbb{Z} / p \mathbb{Z}$.

\subsection{A rank formula}

Before we can apply the rational surgery formula to find the rank of $\widehat{H F}$ of a surgered manifold, we need to first define several invariants pertaining to a chain complex $C F K^{\infty}$. From now on, $Y$ is assumed to be an $\mathrm{L}-$-space homology sphere, unless otherwise specified.

Let $\mathcal{F}_{Y}(K, m):=C\{i=0, j \leq m\} \subset \widehat{C F}(Y)$ be the subcomplex generated by intersection points whose filtration level is less than or equal to $m$. We obtain a sequence of maps

$$
\iota_{K}^{m}: \mathcal{F}_{Y}(K, m) \longrightarrow \widehat{C F}(Y),
$$

which induce isomorphisms in homology for all sufficiently large integers $m$.

Definition 3.3 Define $\tau_{Y}(K)$ by

$\tau_{Y}(K)=\min \{m \in \mathbb{Z} \mid$

$$
\left.\iota_{K}^{m}: \mathcal{F}_{Y}(K, m) \rightarrow \widehat{C F}(Y) \text { induces a nontrivial map in homology }\right\} .
$$

The invariant $\tau_{Y}(K)$ is an invariant of the knot $K$, which gives a lower bound on the four-ball genus of the knot $K$ when $Y=S^{3}$. Likewise, we can define an invariant $v_{Y}(K)$ in a similar manner.

Definition 3.4 Define $v_{Y}(K)$ by

$v_{Y}(K)=\min \left\{s \in \mathbb{Z} \mid \widehat{v}_{s}: \widehat{A}_{s} \rightarrow \widehat{C F}(Y)\right.$ induces a nontrivial map in homology $\}$.

Let $m(K) \subset-Y$ be the mirror image of the knot $K \subset Y$; that is, so to speak, reversing the orientation of the ambient manifold $Y$ while keeping the original orientation of $K$. When $Y=S^{3}, m(K)$ is the mirror image in the usual sense by mirror reflecting $S^{3}$. In general, we claim the identity

$$
\tau_{Y}(K)=-\tau_{-Y}(m(K)) .
$$

whose proof is a direct adaption of [9, Lemma 3.3], following from the duality map $\mathcal{D}: \widehat{C F}_{*}(Y) \rightarrow \widehat{C F}^{*}(-Y)$. 
We also claim that

$$
v_{Y}(K)=\tau_{Y}(K) \text { or } \tau_{Y}(K)+1 .
$$

When $Y=S^{3}$, this is proved in [9, Proposition 3.1] by purely homological-algebra methods. Such an argument would hold for the filtered chain group $C F K^{\infty}(Y, K)$ as well, for $S^{3}$ cannot be distinguished from an arbitrary L-space homology sphere in the chain level.

Therefore, either $v_{Y}(K)$ or $v_{-Y}(m(K))$ is nonnegative.

We are now able to calculate the rank of $\widehat{H F}\left(Y_{p / q}(K)\right)$. Since $\operatorname{rk}\left(Y_{p / q}(K)\right)=$ $\operatorname{rk}\left((-Y)_{-p / q}(m(K))\right.$, it suffices to consider knots $K$ with $v_{Y}(K) \geq v_{-Y}(m(K))$. We obtain the following rank formula analogous to [15, Proposition 9.6].

Proposition 3.5 Let $K$ be a knot in an $L$-space homology sphere $Y$, and fix a pair of relatively prime integers $p$ and $q$ with $p \neq 0$ and $q>0$; and suppose that $v_{Y}(K) \geq v_{-Y}(m(K))$. Then, if $v_{Y}(K)>0$ or $p>0$,

$$
\operatorname{rk}\left(\widehat{H F}\left(Y_{p / q}(K)\right)=p+2 \max \left(0,\left(2 v_{Y}(K)-1\right) q-p\right)+q\left(\sum_{s}\left(\operatorname{rk} H_{*}\left(\widehat{A}_{S}\right)-1\right)\right),\right.
$$

and if $v_{Y}(K)=0$, we have that

$$
\operatorname{rk}\left(\widehat{H F}\left(Y_{p / q}(K)\right)=|p|+q\left(\sum_{s}\left(\operatorname{rk} H_{*}\left(\widehat{A}_{S}\right)-1\right)\right) .\right.
$$

Lemma 3.6 Suppose $K$ is a knot in an L-space homology sphere $Y$, and suppose that $v_{Y}(K)=v_{-Y}(m(K))=0$. Then, the image of

$$
\left(\hat{h}_{0} \oplus \widehat{v}_{0}\right)_{*} \longrightarrow H_{*}(\widehat{B} \oplus \widehat{B}) \cong \mathbb{F} \oplus \mathbb{F}
$$

is one-dimensional.

Proof Let $\delta(K)$ denote the dimension of the image of

$$
\left(\hat{h}_{0} \oplus \widehat{v}_{0}\right)_{*} \longrightarrow H_{*}(\widehat{B} \oplus \widehat{B}) \cong \mathbb{F} \oplus \mathbb{F},
$$

and let $a_{0}(K)$ denote the dimension of $H_{*}\left(\hat{A}_{0}(K)\right)$. Note that both $\delta(K)$ and $\delta(m(K))$ are positive integers by the definition of $v$.

For sufficiently large $N$, we have

$$
\text { rk } \widehat{H F}\left(Y_{N}(K), 0\right)=a_{0}(K) \text {. }
$$

From Theorem 3.2, we have

$$
\operatorname{rk} \widehat{H F}\left(Y_{-N}(K), 0\right)=a_{0}(K)+2-2 \delta(K) .
$$


Similar formulae hold for large $N$ and $-N$ surgeries on $m(K) \subset-Y$. Finally, from the identification $Y_{N}(K)=-(-Y)_{-N}(m(K))$, we conclude that

$$
\begin{aligned}
a_{0}(K) & =a_{0}(m(K))+2-2 \delta(m(K)), \\
a_{0}(m(K)) & =a_{0}(K)+2-2 \delta(K),
\end{aligned}
$$

from which we see $\delta(K)=\delta(m(K))=1$.

Express $\widehat{H F}\left(Y_{p / q}(K)\right)$ in terms of $H_{*}\left(\widehat{\mathbb{X}}_{p / q}\right)$. Proposition 3.5 now follows by the same argument of [15].

\subsection{L-Space surgeries on knots}

Recall that a rational homology three-sphere $Y$ is an $\mathrm{L}$-space if $\widehat{H F}(Y, \mathfrak{s}) \cong \mathbb{Z}$ for each $\operatorname{Spin}^{c}$ structure $\mathfrak{s}$ on $Y$. A knot $K \subset S^{3}$ is said to admit an L-space surgery if certain rational surgery $S_{r}^{3}(K)$ is an L-space. Ozsváth and Szabó's remarkable theorem [13, Theorem 1.2] gives a very strong restriction on the knot Floer homology for such knots.

When $K$ is a knot in an $\mathrm{L}$-space homology sphere $Y$, a similar characterization can be obtained for $\widehat{H F K}(Y, K)$.

Proposition 3.7 Suppose $K \subset Y$ is a knot in an $L$-space homology sphere. If there is a rational number $r$ for which $Y_{r}(K)$ is an $L$-space, then there is an increasing sequence of integers $n_{-k}<\cdots<n_{k}$ with the property that $n_{i}=-n_{-i}$, and $\widehat{H F K}(K, j)=0$ unless $j=n_{i}$ for some $i$, in which case $\widehat{\operatorname{HFK}}(K, j) \cong \mathbb{Z}_{\left(\delta_{i}\right)}$, supported in dimension $\delta_{i}$ that are alternating in parities.

Proof Observe that the original proof in [13, Theorem 1.2] for $S^{3}$ utilizes only the information of the filtered chain. So the entire argument can be carried through here without any change.

Taking the Euler characteristic of the knot Floer homology, we obtain the following restriction on Alexander polynomials, particularly convenient for our purpose.

Corollary 3.8 Let $K$ be a knot that admits an L-space surgery. Then, the Alexander polynomial of $K$ has the form

$$
\Delta_{K}(T)=(-1)^{k}+\sum_{j=1}^{k}(-1)^{k-j}\left(T^{n_{j}}+T^{-n_{j}}\right),
$$

for some increasing sequence of positive integers $0<n_{1}<n_{2}<\cdots<n_{k}$. 


\section{Proof of the main results}

Proof of Theorems 1.2 and 1.4 Suppose there were truly cosmetic surgeries for a nontrivial knot $K$ in an integral homology sphere $Y$. Since for the surgered manifold, $H_{1}\left(Y_{p / q}(K)\right)=\mathbb{Z} / p \mathbb{Z}$, it suffices the consider the case $Y_{p / q}(K) \cong Y_{p / q^{\prime}}(K)$. (The $\infty$ surgery can be treated as an $1 / 0$ surgery.) Comparing their Casson-Walker invariants and Casson-Gordon invariants and resorting to the surgery formulae (1), (2) gives

$$
\begin{aligned}
\lambda(Y)+\lambda(L(p, q))-\frac{q}{p} \Delta_{K}^{\prime \prime}(1) & =\lambda(Y)+\lambda\left(L\left(p, q^{\prime}\right)\right)-\frac{q^{\prime}}{p} \Delta_{K}^{\prime \prime}(1), \\
\tau(L(p, q))-\sigma(K, p) & =\tau\left(L\left(p, q^{\prime}\right)\right)-\sigma(K, p),
\end{aligned}
$$

and we conclude $Y_{p / q}(K) \not Y_{p / q^{\prime}}(K)$ provided $\Delta_{K}^{\prime \prime}(1) \neq 0$. See [1, Proposition 5.1]. We shall see that the Heegaard Floer homology supplies exactly the missing piece. Suppose $q$ and $q^{\prime}$ have the same sign. Proposition 3.5 implies that $\operatorname{rk}\left(\hat{A}_{S}\right)=1$ for all $s$, and $\operatorname{rk} \widehat{H F}\left(Y_{p / q}(K)\right)=\operatorname{rk} \widehat{H F}\left(Y_{p / q^{\prime}}(K)\right)=p$. Hence, $K$ is a knot that admits an L-space surgery, and consequently $\Delta_{K}(T)=(-1)^{k}+\sum_{j=1}^{k}(-1)^{k-j}\left(T^{n_{j}}+T^{-n_{j}}\right)$ by Corollary 3.8.

We claim that $\Delta_{K}^{\prime \prime}(1) \neq 0$ unless $\Delta_{K}(T)=1$. This follows from a straightforward computation

$$
\Delta_{K}^{\prime \prime}(1)=2 \sum_{j=1}^{k}(-1)^{k-j} n_{j}^{2}
$$

and the fact that $0<n_{1}<n_{2}<\cdots<n_{k}$.

For those knots $K$ with $\Delta_{K}(T)=1$, Proposition 3.7 implies, in addition, that $\widehat{H F K}(K, 0)=\mathbb{Z}$, and $\widehat{H F K}(K, j)=0$ for any other $j$. Hence $K=U$, by the fact that knot Floer homology detects the unknot [8]. This finishes the proof of the theorems.

\section{References}

[1] S Boyer, D Lines, Surgery formulae for Casson's invariant and extensions to homology lens spaces, J. Reine Angew. Math. 405 (1990) 181-220 MR1041002

[2] A J Casson, C M Gordon, On slice knots in dimension three, from: "Algebraic and geometric topology (Proc. Sympos. Pure Math., Stanford Univ., 1976), Part 2", (R J Milgram, editor), Proc. Sympos. Pure Math. XXXII, Amer. Math. Soc. (1978) 39-53 MR520521

[3] C M Gordon, J Luecke, Knots are determined by their complements, J. Amer. Math. Soc. 2 (1989) 371-415 MR965210 
[4] R Kirby, editor, Problems in low-dimensional topology, from: "Geometric topology (Athens, GA, 1993)”, AMS/IP Stud. Adv. Math. 2, Amer. Math. Soc. (1997) 35-473 MR1470751

[5] P Kronheimer, T Mrowka, P Ozsváth, Z Szabó, Monopoles and lens space surgeries, Ann. of Math. (2) 165 (2007) 457-546 MR2299739

[6] M Lackenby, Dehn surgery on knots in 3-manifolds, J. Amer. Math. Soc. 10 (1997) 835-864 MR1443548

[7] Y Ni, Non-separating spheres and twisted Heegaard Floer homology arXiv: 0902.4034

[8] Y Ni, Link Floer homology detects the Thurston norm, Geom. Topol. 13 (2009) 29913019 MR2546619

[9] P Ozsváth, Z Szabó, Knot Floer homology and the four-ball genus, Geom. Topol. 7 (2003) 615-639 MR2026543

[10] P Ozsváth, Z Szabó, Holomorphic disks and knot invariants, Adv. Math. 186 (2004) 58-116 MR2065507

[11] P Ozsváth, Z Szabó, Holomorphic disks and three-manifold invariants: properties and applications, Ann. of Math. (2) 159 (2004) 1159-1245 MR2113020

[12] P Ozsváth, Z Szabó, Holomorphic disks and topological invariants for closed threemanifolds, Ann. of Math. (2) 159 (2004) 1027-1158 MR2113019

[13] P Ozsváth, Z Szabó, On knot Floer homology and lens space surgeries, Topology 44 (2005) 1281-1300 MR2168576

[14] P Ozsváth, Z Szabó, Knot Floer homology and integer surgeries, Algebr. Geom. Topol. 8 (2008) 101-153 MR2377279

[15] P Ozsváth, Z Szabó, Knot Floer homology and rational surgeries, Algebr. Geom. Topol. 11 (2011) 1-68 MR2764036

[16] J Rasmussen, Floer homology and knot complements, $\mathrm{PhD}$ thesis, Harvard University (2003) arXiv:math/0306378

[17] K Walker, An extension of Casson's invariant, Annals of Math. Studies 126, Princeton Univ. Press (1992) MR1154798

[18] J Wang, Cosmetic surgeries on genus one knots, Algebr. Geom. Topol. 6 (2006) 14911517 MR2253457

Department of Mathematics, California Institute of Technology

Pasadena CA 91125, USA

zhongtao@caltech.edu

http://www.its.caltech.edu/ zhongtao/

Proposed: Rob Kirby

Seconded: Danny Calegari, Joan Birman
Received: 4 October 2010

Revised: 11 April 2011 